\title{
The design of the botanical indoor air biofilter system for the atmospheric particle removal
}

\author{
Izdihar Zahirah Ibrahim ${ }^{1, *}$, Wen-Tong Chong ${ }^{1}$, and Sumiani Yusoff ${ }^{2}$ \\ ${ }^{1}$ Department of Mechanical Engineering, Faculty of Engineering, University of Malaya, 50603 Kuala Lumpur, Malaysia \\ ${ }^{2}$ Department of Civil Engineering, Faculty of Engineering, University of Malaya, 50603 Kuala Lumpur, Malaysia
}

\begin{abstract}
Indoor environmental quality (IEQ) objective generally focus on providing energizing and comfortable environments for occupants and minimizing the risk of building-related health problems. Living green walls are natural air-filters that creates a cleaner and revitalizing work environment that will lead to better IEQ. The research presented here describes the design (the new concept) of the botanical indoor air biofilter (BIAB) and modelling conducted to determine the effectiveness of the system in reducing the indoor airborne particulate matter levels. The BIAB was also evaluated for its single-pass filtration for particles ranging in diameter from 2.5 to $10 \mu \mathrm{m}$ along with total suspended particles. The system is comprised of three functional components; a region of vertically grown plants as botanical section, an evaporative cooling pad as cooling section (additional section from a commercial BIAB), and a mechanical ventilation system that supply cool filtered air to surrounding. The complete system recorded highest removal efficiencies of $85 \%$ for TSP, $75.2 \%$ for $\mathrm{PM}_{2.5}$, and $71.9 \%$ for $\mathrm{PM}_{10}$. It indicated that with the additional component in the BIAB system (cooling component), it provides enhancement of the particulate removal due to the ability in absorbing the dust particles and filtration dynamics as the polluted air pass through the wetted cooling pad and the light shower of water.
\end{abstract}

\section{Introduction}

People are frequently concerned that they will have unhealthy symptoms or health conditions from exposures to the contaminants in the buildings where they spend time for a long period. People's satisfaction with the indoor environmental quality (IEQ) is important because it influences productivity and health [1]. Indoor air quality and thermal comfort are the important main subjects to consider of living indoor [2]. The combination of high temperature and high relative humidity serves to reduce thermal comfort and indoor air quality [3]. Most of the people who works in the office within 8 hours are more productive when they are satisfied with their indoor environment [4].

Thus, to maintain the comfort of the indoor environment, many buildings normally use an air conditioning and mechanical ventilation (ACMV) system. This solution requires high levels of energy consumption and also contribute to one of a major environmental issue, such as increasing emissions of greenhouse gases to the atmosphere that causes global warming. In addition, even in buildings with efficient ACMV systems, fine particulate matter (PM) might infiltrate into indoor environments as it cannot be effectively filtered out of the incoming air [5]. Indeed, the indoor air quality are affected from the concentration of outdoor pollutants and most of the large proportion of the indoor particles are from outdoor [6]. In addition, even with the filtration process from the portable indoor air filters, it cannot ensure the effectiveness of the particle removal will stand for a long period [7].

In this circumstance, the practice of the indoor greening system alone or in combination with conventional ACMV systems in buildings may provide environmental value and in addition contribute to reduce energy consumption [8]. By employing a greening system in the interior building, the effect on indoor air temperature and humidity are significant [9]. In the trend of indoor greening, green wall technology has led to the development of activated systems, termed botanical biofilters, that move air through the plant growth substrate to increase the rate at which the interior atmospheric environment is exposed to the components of the plantsubstrate system that are active in air pollutant removal [10]. The majority of research that has been conducted on green wall had focused on defining it as botanical indoor air biofilter (BIAB) that can remove $\mathrm{VOC}, \mathrm{CO} 2$ and particulate matter [10-13]. For example, Irga et al. [10] determined the effectiveness of BIAB in reducing $\mathrm{PM}_{2.5}$ and PM10 that evaluated with single-pass filtration efficiency and compare with portable in-room air cleaner.

The research presented here describes the design (the new concept) of the botanical indoor air biofilter (BIAB) and modelling conducted to determine the effectiveness of the system in reducing particulate matter levels. The $\mathrm{BIAB}$ was also evaluated for its single-pass filtration for particles ranging in diameter from 2.5 to $10 \mu \mathrm{m}$ along with total suspended particles. 


\section{BIAB system design}

The study uses a new concept of a mini type active green wall (desktop size), see Fig. 1. Briefly, the system is comprised of three functional components; a region of vertically grown plants as botanical section, an evaporative cooling pad as cooling section (additional section from a commercial BIAB), and a mechanical ventilation system that supply cool filtered air to surrounding. The system consists of a $0.05 \mathrm{~m}^{3}(38 \mathrm{x} 38 \mathrm{x}$ $34 \mathrm{~cm}$ ) polyethylene module with 9 holes on the front face which plants grow horizontally from the circular compartment in the casing. Each hole in the module has $27.3 \%$ of the front face allowing air to pass. For these experiments, each circular compartment in the hole contains Epipremnum aureum (Golden photos) as the botanical component and Kenaf fibre as growth medium in the system. The cooling sections in these system uses a corrugated paper type of cooling pad $\left(1.2 \mathrm{~m}^{2}\right.$ with $5 \mathrm{~cm}$ thickness) and a submersible mechanical water pump ( 8 watt) to wetted the cooling pad, at the same time function as water irrigation system in the module. At the back face of the module consists 8 no. of axial brushless fan (DC 12 -volt, 1.44 watt in $80 \times 80 \times 15 \mathrm{~mm}$ casing size) with total airflow rate $150 \mathrm{~L} . \mathrm{s}^{-1}$ and function to draw air from the indoor space through the botanical component, within the growth medium, cross the moistened pad and back into indoor surrounding.
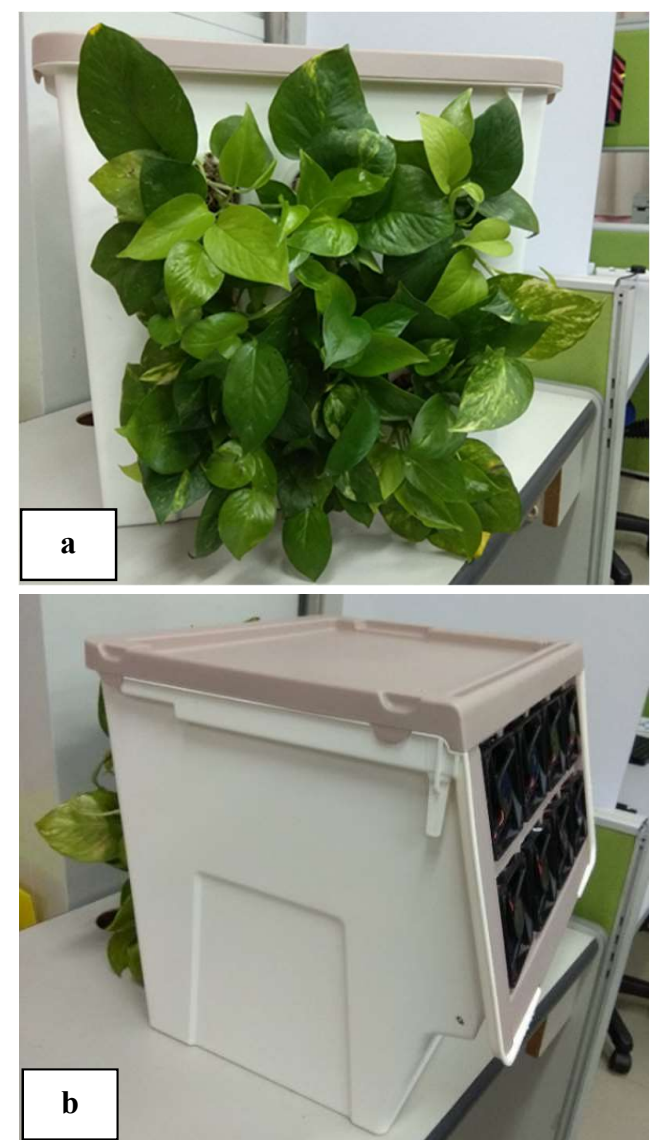

Fig. 1. (a) The front view of BIAB system module; (b) the side view.

\section{Methodology}

\subsection{Single pass efficiency test of the BIAB}

The study described by Irga et al. [14], sealed tight acrylic Perspex test chamber $(0.6$ x $0.6 \times 0.6 \mathrm{~m} ; 216 \mathrm{~L}$ with 10 $\mathrm{mm}$ thk.) was used in these experiments. At the one side of the chamber was designed to be a detachable door (sealed with adhesive foam rubber and adjustable metal clamps) to allow the placement of the module into the chamber and at the opposite side, flexible ducting $(13 \mathrm{~cm}$ diameter) was fixed to the centre of the chamber (see Fig.2). The fitted ducting led to a combustion chamber in which PM was generated by burning an 80 -gsm square paper $(210 \times 297 \mathrm{~mm})$ with a $4 \mathrm{~mL}$ of filtered retail-grade diesel fuel (Petronas).

The generated PM flowed through the fitted ducting with active airflow provided by an axial brushless fan (DC 12-volt, 1.8 watt in $120 \times 120 \times 25 \mathrm{~mm}$ casing size) housed in the centre of the chamber (fitted with the ducting), before flowing through the BIAB module where pollutant air is distributed through the front face of the module (botanical section). A fan within the test chamber helped to spreading of the filtered airflow throughout the chamber to reduce precipitation of particles before exhaust into another ducting system (vacuum exhaust) that fixed to the detachable door of the chamber, which led to a tube of laser nephelometer (TSI Dustrak II 8531) to record average particle density and size distribution of the filtered airstream. After each sampling, the air was exhausted to spare via a vacuum exhaust pump.

To compare the efficiency of the system, the experiment was conducted in three different variations; firstly, to test a new concept of BIAB complete system (botanical and cooling component), secondly to test only cooling component without botanical component and lastly to test only botanical component without cooling component. For each variation, average PM concentration was recorded for three particle sizes; total suspended particle (TSP), $\mathrm{PM}_{2.5}$ and $\mathrm{PM}_{10}$. Duplicate tests were conducted for each experimental condition and all of the replicate trials were recorded for $10 \mathrm{~min}$, which was sufficient time for the PM concentration to return to ambient levels for all treatments.

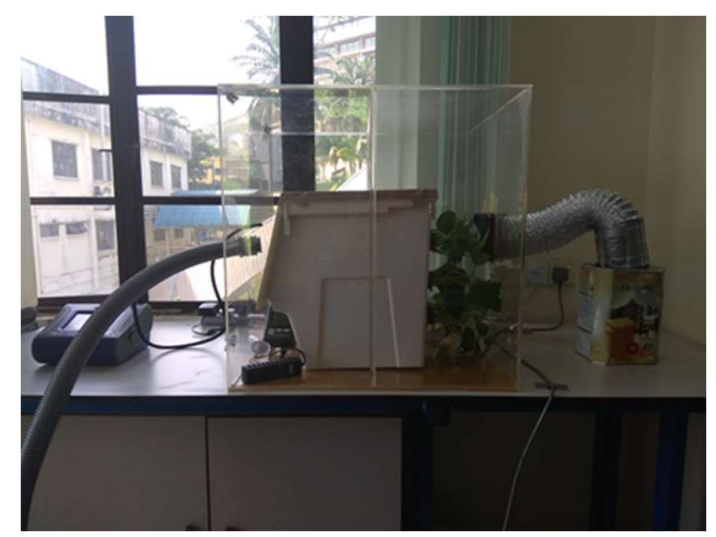

Fig. 2. Photograph depicting experimental testing apparatus used to expose the modular botanical air biofilter to a single pulse event of particular matter. 


\subsection{Data analysis}

The analysis presented by Irga et al. [10], real-time concentration data from each single pass efficiency test was used to construct concentration versus time profiles. In order to account for the differences in pressure created by the different treatments, as well as the residence time distribution (RTD) differences (which is the amount of time a pollutant that is not filtered spends within the system until reaching the detector), the area under the concentration versus time data was calculated and subsequently used as the dependent variable.

Single pass filtration efficiency $(\eta)$ was calculated using Equations (1) [15]:

$$
\eta=\left(\frac{C_{\text {in }}-C_{\text {out }}}{C_{\text {in }}}\right) \times 100 \%
$$

where:

$\mathrm{C}_{\text {in }}=$ integration of the average particle concentration decay curve in the duct with no application of treatment
$\mathrm{C}_{\text {out }}=$ integration of the average particle concentration decay curve in the duct with application of treatment

Particle resolved CADR derived from the single pass efficiency data CADR was calculated utilizing Equation (2) $[15]:$

$$
C A D R=\eta \times G
$$

where:

$\eta=$ the single pass efficiency of the system $\mathrm{G}=$ airflow rate through the system $\left[\mathrm{L} . \mathrm{s}^{-1}\right]$

\section{Result and discussion}

Single pass removal efficiencies for three particle sizes; total suspended particle (TSP), $\mathrm{PM}_{2.5}$ and $\mathrm{PM}_{10}$ with across three different variances are shown in Fig. 3, and a comparative summary of treatment CADR values derived from $\eta$ for the particle fractions measured is presented in Table 1.

\section{complete system cooling component only $\square$ botanical component only}

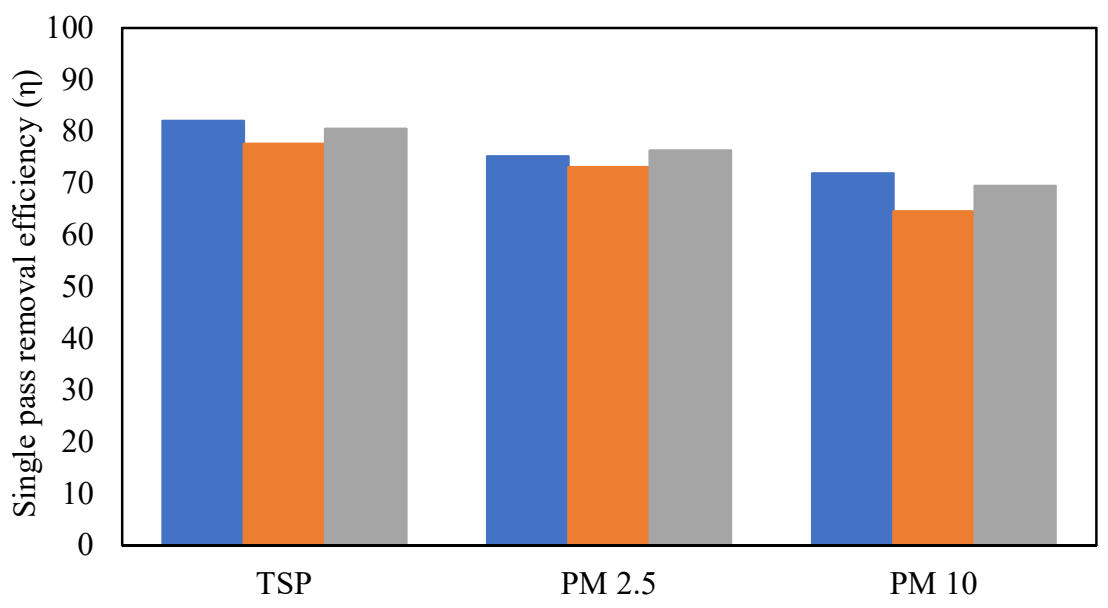

Fig. 3. Calculated single-pass removal efficiency $(\eta)$ for TSP, $\mathrm{PM}_{2.5}$ and $\mathrm{PM}_{10}$.

Table 1. CADR $\left(L \cdot s^{-1}\right)$ calculated from single pass efficiency tests, when air flow is set to maximum.

\begin{tabular}{llll}
\hline Pollutant & Complete system & Cooling component only & Botanical component only \\
\hline TSP & 123.00 & 116.40 & 120.72 \\
$\mathrm{PM}_{2.5}$ & 112.80 & 109.66 & 114.52 \\
$\mathrm{PM}_{10}$ & 107.88 & 96.86 & 104.17 \\
\hline
\end{tabular}


Removal was achieved in all treatments, and it can be seen that there were strong similarities in response across the three particle sizes tested. As expected, the new concept of BIAB complete system module treatment showed the highest removal rates, demonstrating removal efficiencies of $85 \%$ for TSP, $75.2 \%$. for $\mathrm{PM}_{2.5}$, and $71.9 \%$ for $\mathrm{PM}_{10}$. Comparatively, the system that tested only with cooling section and without the botanical component present recorded removal efficiencies of $77.6 \%$ for TSP, $73.1 \%$ for $\mathrm{PM}_{2.5}$, and $64.57 \%$ for $\mathrm{PM}_{10}$, which were significantly lower than the removal efficiencies recorded for the complete system module. Additionally, when the system was tested with only botanical component present and without cooling component, removal efficiencies were $80.48 \%$ for TSP, $76.3 \%$ for $\mathrm{PM}_{2.5}$, and $69.4 \%$ for $\mathrm{PM}_{10}$. This study provides data for the characterisation of the removal efficiency of atmospheric particles by a new concept of botanical indoor air biofiltration (BIAB) system. We state that a higher removal of particulates from air can be achieved by ventilation of the polluted airstream through the two stages of the particle removal (botanical and cooling component) by the BIAB tested.

Recent study of active living wall focusing on the effect of PM removal efficiency, Irga et al. [10] is the first to document the CADR value for PM. The system is slightly different to the system presented here, as it consists of plant with packing media (plant-substrate system), where the air move through the plant growth substrate to increase the rate of PM removal efficiency. Chlorophytum comosum (Spider plant) being used as the botanical component in that system. Irga et al. [10] demonstrated that their system had removal efficiencies $53.35 \pm 9.73 \%$ for TSP, $53.51 \pm 15.99 \%$ for $\mathrm{PM}_{10}$, and $48.21 \pm 14.71 \%$ for $\mathrm{PM}_{2.5}$, which were significantly lower than the removal efficiencies recorded for the HVAC filter at an air flow rate of $11.25 \mathrm{~L} \mathrm{~s}^{-1}$. Additionally, Irga et al. [19] defined the CADR value of their ALW slightly less efficient than HVAC filter, with a total value of 28.70 $\mathrm{m}^{3} \mathrm{~h}^{-1}$ for $\mathrm{PM}_{10}$ and $25.86 \mathrm{~m}^{3} \mathrm{~h}^{-1}$ for $\mathrm{PM}_{2.5}$.

Comparatively, the removal efficiencies recorded here are significantly higher than the results in Irga et al. [10], the experimented BIAB system have a higher airflow rate than their tested botanical biofilter module. Furthermore, this could be due to the additional cooling component inside the system and plant species differences. The PM real-time reduction appears likely that the additional cooling component in the system has an effect on absorbing the dust particles and filtration dynamics as the polluted air pass through the wetted cooling pad and the light shower of water.

\section{Conclusion}

The research presented here quantifies the additional functionality (new concept) of a botanical indoor air biofilter system, with regards to the removal of particulate matter from air. The complete system recorded highest removal efficiencies of $85 \%$ for TSP, $75.2 \%$. for $\mathrm{PM}_{2.5}$, and $71.9 \%$ for $\mathrm{PM}_{10}$. It indicated that with the additional component in the BIAB system (cooling component), it provides enhancement of the particulate removal due to the ability in absorbing the dust particles and filtration dynamics as the polluted air pass through the wetted cooling pad and the light shower of water. The future studies are to focus on system's ability to remove VOCs and $\mathrm{CO}_{2}$ and improve temperature and humidity of the indoor environment. Nonetheless, further controlled laboratory experiments are needed to investigate the longterm performance of the system, and to better describe the simultaneous removal of PMs, VOCs and $\mathrm{CO}_{2}$. These investigations will provide empirical data on which to develop a simulation model that can be used to optimize the system's design, as well as to advance the implementation of the device.

The authors would like to thank the University of Malaya for the research grants allocated under the Sustainability Science (SuSci) Research Cluster, UM Living Lab Grant Programme (LL019-16-SUS)

\section{References}

[1] S. Kang, D. Ou, C.M. Mak, The impact of indoor environmenta quality on work productivity in university open-plan research offices, Building and Environment (2017).

[2] J. Kim, T. Hong, J. Jeong, C. Koo, M. Kong, An integrated psychological response score of the occupants based on their activities and the indoor environmental quality condition changes, Building and Environment 123 (2017) 66-77.

[3] L. Fang, D.P. Wyon, G. Clausen, P.O. Fanger, Impact of indoor air temperature and humidity in an office on perceived air quality, SBS symptoms and performance, Indoor Air 14 Suppl 7 (2004) 74-81.

[4] C. Huizenga, S. Abbaszadeh, L. Zagreus, E.A. Arens, Air quality and thermal comfort in office buildings: Results of a large indoor environmental quality survey, 2006.

[5] R.B. Mosley, D.J. Greenwell, L.E. Sparks, Z. Guo, W.G. Tucker, R Fortmann, C. Whitfield, Penetration of Ambient Fine Particles into the Indoor Environment, Aerosol Science and Technology 34(1) (2001) 127-136.

[6] R. Meier, C. Schindler, M. Eeftens, I. Aguilera, R.E. Ducret-Stich, A. Ineichen, M. Davey, H.C. Phuleria, N. Probst-Hensch, M.Y. Tsai, N. Kunzli, Modeling indoor air pollution of outdoor origin in homes of SAPALDIA subjects in Switzerland, Environ Int 82 (2015) 85-91.

[7] M.S. Zuraimi, M. Vuotari, G. Nilsson, R. Magee, B. Kemery, C. Alliston, Impact of dust loading on long term portable air cleaner performance, Building and Environment 112 (2017) 261-269.

[8] B. Raji, M.J. Tenpierik, A. van den Dobbelsteen, The impact of greening systems on building energy performance: A literature review, Renewable and Sustainable Energy Reviews 45 (2015) 610-623.

[9] D. Tudiwer, A. Korjenic, The effect of an indoor living wall system on humidity, mould spores and $\mathrm{CO} 2$-concentration, Energy and Buildings 146 (2017) 73-86.

[10] P.J. Irga, N.J. Paull, P. Abdo, F.R. Torpy, An assessment of the atmospheric particle removal efficiency of an in-room botanical biofilter system, Building and Environment 115 (2017) 281-290.

[11] Z. Wang, J.S. Zhang, Characterization and performance evaluation of a full-scale activated carbon-based dynamic botanical air filtration system for improving indoor air quality, Building and Environment 46(3) (2011) 758-768

[12] A.B. Darlington, J.F. Dat, M.A. Dixon, The Biofiltration of Indoor Air: Air Flux and Temperature Influences the Removal of Toluene, Ethylbenzene, and Xylene, Environmental Science \& Technology 35(1) (2001) 240-246.

[13] D. Llewellyn, M. Dixon, 4.26 - Can Plants Really Improve Indoor Air Quality?, in: M. Moo-Young (Ed.), Comprehensive Biotechnology (Second Edition), Academic Press, Burlington, 2011, pp. 331-338. [14] T. Pettit, P.J. Irga, P. Abdo, F.R. Torpy, Do the plants in functional green walls contribute to their ability to filter particulate matter?, Building and Environment 125(Supplement C) (2017) 299-307.

[15] U.S. EPA, Evaluation of In-Room Particulate Matter Air Filtration Devices, U.S. Environmental Protection Agency, Washington, DC, 2008.

\footnotetext{
* Corresponding author: izdiharzahirah@gmail.com
} 